\title{
Mortalidad infantil y marginación urbana: análisis espacial de su relación en una ciudad de tamaño medio del noroeste mexicano
}

\author{
Gerardo Álvarez, ${ }^{1}$ Francisco Lara, ${ }^{2}$ Siobán D. Harlow ${ }^{3}$ y Catalina Denman ${ }^{4}$
}

Forma de citar

Álvarez G, Lara F, Harlow SD, Denman C. Mortalidad infantil y marginación urbana: análisis espacial de su relación en una ciudad de tamaño medio del noroeste mexicano. Rev Panam Salud Publica. 2009;26(1):31-8.

RESUMEN Objetivo. Identificar las áreas de alto riesgo de mortalidad infantil y su posible correlación con el nivel socioeconómico de una población, mediante la combinación de un sistema de información geográfica y técnicas de análisis espacial.

Métodos. Estudio exploratorio ecológico realizado en Hermosillo, capital de Sonora, México, en 2000-2003. Para cada área geoestadística básica (AGEB) de la ciudad se calcularon el índice de marginación urbana (IMU) y la tasa de mortalidad infantil (TMI). Los IMU y las TMI se procesaron estadísticamente para identificar los puntos de concentración geográfica y determinar el grado de correlación espacial entre ambos indicadores. Para evaluar la autocorrelación espacial general y los agrupamientos espaciales de las TMI y los IMU en la ciudad y sus AGEB se emplearon el indice de Moran I, el estadístico Ipop y el método de Besag y Newell.

Resultados. La TMI promedio fue de 14,3 por 1000 nacidos vivos, mayor en las AGEB con alto nivel de marginación social (16,2 por 1000$)$ y menor en las de bajo nivel (11,7 por 1000$)$. El IMU en los AGEB varió entre -3,1 y 6,6. Se encontró autocorrelación en los IMU (Moran $I=0,62)$, con agrupamientos significativos en el noroeste, noreste y suroeste de la ciudad. Se observaron agrupamientos locales de elevada TMI en las zonas central y occidental de Hermosillo, aunque sin autocorrelación (Moran $I=-0,007$ ). Se identificaron áreas de alto riesgo (altas TMI y altos IMU) en el noroeste de la ciudad.

Conclusión. Se encontraron agrupamientos espaciales con altas TMI en áreas socialmente marginadas del noroeste de Hermosillo, una ciudad de tamaño medio ubicada en el noroeste de México. Estos resultados, obtenidos mediante la combinación de técnicas de análisis espacial y herramientas de los SIG pueden ayudar a dirigir intervenciones específicas hacia esas áreas residenciales de alto riesgo.

Palabras clave Mortalidad infantil, aislamiento social, localización geográfica de riesgo, México.

1 Departamento de Medicina y Ciencias de la Salud, Universidad de Sonora, Hermosillo, Sonora, México. La correspondencia se debe dirigir a Gerardo Álvarez, Blvd. Colosio entre Reforma y Francisco Salazar, Ed. 7C, Colonia Centro, Hermosillo, Sonora CP 83200, México. Correo electrónico: galvarez@ guayacan.uson.mx

2 Escuela de Planeamiento, Universidad Estatal de Arizona, Tempe, Arizona, Estados Unidos de América.

3 Escuela de Salud Pública, Universidad de Michigan, Ann Arbor, Michigan, Estados Unidos de América.

4 Programa Salud y Sociedad, El Colegio de Sonora, Hermosillo, Sonora, México.
A partir de la segunda mitad del siglo $X X$, las tasas de mortalidad infantil (TMI) descendieron en forma consistente y rápida en México. Entre 1970 y 2005, la tasa pasó de 80,0 a 16,6 por 1000 nacidos vivos (1) como resultado de cambios en la estructura social y económica del país. También influyeron el fortalecimiento de las instituciones de salud y la implementación de estrategias exitosas de salud pública, como las campañas masivas de inmunización, la mejoría nutricional y el tratamiento adecuado de las neumonías y las diarreas (2). No obstante, aún persisten marcadas diferencias sociales y regionales en la distribución de las TMI a lo largo del país y esta es aún alta en comparación con los estándares internacionales (3).

En algunos centros urbanos del noroeste de México se han encontrado TMI muy elevadas (4). Algunas ciudades de 
esta región mexicana han crecido aceleradamente como resultado del proceso de reestructuración económica que desde mediados de la década de 1990 impulsó la inversión extranjera y el desarrollo de la industria maquiladora, además del desplazamiento de la población rural y semiurbana hacia las ciudades. Esa reestructuración ha provocado una reducción del gasto público en saneamiento, agua potable, vivienda, infraestructura y otros servicios municipales (5). En consecuencia, la percepción de que las condiciones de vida se han deteriorado en las ciudades del norte de México y que solo algunos reciben los beneficios de la vida urbana son elementos frecuentes en estudios que han documentado desigualdades sociales en las ciudades de esta región (6-7). Esta situación ha generado disparidades que explican las diferencias observadas en las tasas de morbilidad y mortalidad de grupos de población más o menos privilegiados (8-9).

El reconocimiento de las diferencias sociales y regionales en la distribución de la mortalidad infantil es un elemento crítico para la formulación de políticas de salud eficaces. La mortalidad infantil no es un fenómeno aleatorio, ya que presenta variaciones regionales que en gran medida son consecuencia de las desigualdades en salud y guardan relación directa con diversos factores socioeconómicos (10-11). En este sentido, cobran particular relevancia los esfuerzos que se vienen realizando desde hace décadas para examinar la relación de algunos indicadores poblacionales del nivel socioeconómico con la distribución de la mortalidad infantil (12-16).

A fin de comprender mejor la distribución geográfica de la mortalidad infantil y su asociación con diversos factores, se han desarrollado técnicas e indicadores específicos que permiten identificar y caracterizar sus patrones espaciales y estimar la variación geográfica de su incidencia. Uno de esos indicadores es el índice de Moran I, que describe si áreas residenciales contiguas tienen valores similares de un evento de interés y, por lo tanto, si su posible agrupamiento espacial no es resultado de un fenómeno aleatorio (17). Otro indicador de la posible correlación territorial entre dos variables es el índice local bifactorial de asociación espacial, que refleja el grado de asociación lineal (positiva o negativa) entre el valor de una variable en una locación dada y el promedio de otra varia- ble en locaciones vecinas (18). La combinación de estas herramientas de análisis espacial con datos obtenidos mediante sistemas de información geográfica (SIG) ha demostrado su utilidad para el estudio de la distribución de la mortalidad infantil $(10,19)$.

Sin embargo, a pesar de su enorme potencial en el campo de la salud pública, los SIG y las técnicas de análisis espacial no han recibido la suficiente atención. La aplicación combinada de estas metodologías puede contribuir a localizar los factores de riesgo y los eventos relacionados con el binomio salud-enfermedad a escala de barrio y otras subdivisiones territoriales de las ciudades.

El objetivo del presente estudio fue identificar las áreas de alto riesgo de mortalidad infantil y su posible correlación con el nivel socioeconómico de una población en una ciudad del noroeste mexicano mediante la combinación de un SIG y técnicas de análisis espacial.

\section{MATERIALES Y MÉTODOS}

Se realizó un estudio exploratorio ecológico de la distribución espacial del nivel de marginación social y las TMI en Hermosillo, capital del estado de Sonora, en el noroeste de México, en el período 2000-2003. Como unidad de análisis se utilizaron las áreas geoestadísticas básicas $(\mathrm{AGEB})^{5}$ de la ciudad. Para cada AGEB se calcularon el índice de marginación urbana (IMU), elaborado a partir del nivel socioeconómico de sus habitantes, y la TMI. Para determinar el exceso de riesgo en un bloque censal se utilizó la razón de la TMI específica de ese AGEB entre la TMI observada en el área de menor TMI de la ciudad. Tanto los IMU como las TMI se procesaron estadísticamente para identificar los puntos de concentración geográfica y determinar el grado de correlación espacial entre ambos indicadores.

La ciudad de Hermosillo, con una población de 641791 habitantes en 2005 (20), es un centro urbano en proceso de

\footnotetext{
5 Las áreas geoestadísticas básicas (AGEB) son unidades territoriales utilizadas en el levantamiento censal mexicano por el Instituto Nacional de Estadística, Geografía e Informática (INEGI). En general, una AGEB incluye de 25 a 50 manzanas urbanas con una población promedio de 2500 habitantes. En la delineación de una AGEB se toman en cuenta las características demográficas, el nivel económico de la población residente y las condiciones de las viviendas, lo que permite conformar unidades territoriales relativamente homogéneas.
}

expansión como resultado de la localización de compañías transnacionales atraídas por el costo relativamente bajo de la mano de obra, la abundancia de suelo para uso industrial, la cercanía a la frontera con los Estados Unidos de América y los beneficios del Tratado de Libre Comercio de América del Norte. En términos poblacionales, Hermosillo es la tercera ciudad en importancia del noroeste mexicano. Según los resultados del Censo Nacional de Población y Vivienda del año 2000 (21), la ciudad tiene cerca de 133283 viviendas habitadas, distribuidas en más de 370 colonias (barrios) y fraccionamientos (unidades habitacionales), agrupados en 254 AGEB.

\section{Datos e indicadores}

Los datos de mortalidad infantil se obtuvieron del Sistema Epidemiológico y Estadístico de Defunciones, una base de datos digital integrada y controlada por la Secretaría de Salud Pública del Estado de Sonora. Los datos de mortalidad de ese sistema se toman de los certificados de defunción elaborados por la Oficialía del Registro Civil del Estado de Sonora. En el estudio se tomaron en cuenta solamente las muertes de niños y niñas menores de 1 año ocurridas en el área urbana del municipio de Hermosillo entre el 1 de enero de 2000 y el 31 de diciembre de 2003. Cada muerte registrada se vinculó geográficamente al AGEB de la última residencia de la madre. De 702 certificados de defunción examinados, 36 $(5,1 \%)$ no tenían información suficiente para asignar con exactitud el AGEB de residencia, por lo que en esos casos se utilizó la metodología propuesta por Hijar y colaboradores (22) para establecer la referencia geográfica. El número de nacidos vivos por AGEB, según los mismos criterios utilizados para la localización geográfica de las muertes infantiles, se obtuvo de una base de datos proporcionada por la Oficialía del Registro Civil estatal conformada a partir de los certificados de nacimiento. El procedimiento utilizado permitió garantizar la confidencialidad de la información, ya que ninguna de las muertes o nacimientos se vinculó a un domicilio determinado.

Para determinar el nivel socioeconómico de cada AGEB se utilizó el IMU, ampliamente empleado en México para identificar comunidades con carencias socioeconómicas (23). Este índice se determinó mediante la técnica de compo- 
nentes principales a partir de datos socioeconómicos del Censo Nacional de Población y Vivienda del año 2000 (21), entre ellos, la escolaridad, el ingreso familiar, las viviendas con hacinamiento o construidas con materiales precarios, la posesión de automóvil y la tenencia de seguro de salud. Cada AGEB se asignó a alguna de las siguientes categorías de marginación social: muy alta (mayor índice de marginación), alta, media, baja y muy baja (menor índice de marginación).

\section{Análisis de los datos}

Las TMI y los IMU se introdujeron en un SIG elaborado a partir de los mapas urbanos de Hermosillo creados para el levantamiento censal del año 2000. El análisis geográfico se hizo mediante el programa ArcMap (ESRI, Redlands, CA, Estados Unidos) y se calcularon los centroides, se realizó la geocodificación y se elaboraron mapas temáticos, tanto para la mortalidad infantil como para los niveles de marginación social.

Para evaluar la autocorrelación espacial general de las TMI y los IMU en la ciudad y sus AGEB se empleó el índice de Moran I, en el que un valor mayor de cero indica que existe una autocorrelación espacial positiva, es decir, que áreas cercanas presentan tasas similares del evento; mientras un valor cercano a 1 indica un patrón agrupado, un valor cercano a -1 indica que el patrón es aleatorio y un valor de cero indica que no hay correlación espacial (17).

Debido a que se esperaba cierto grado de heterogeneidad en la distribución geográfica de las TMI, se utilizó el estadístico Ipop de Oden para ajustar la influencia que ejercería el tamaño poblacional sobre la probabilidad del agrupamiento espacial general (24). El valor del estadístico Ipop es mayor cuando hay agrupamientos espaciales dentro de una región o entre regiones adyacentes, y se aproxima a cero cuando no hay agrupamiento.

Además, con el propósito de identificar agrupamientos espaciales locales en las AGEB urbanas se empleó el método de Besag y Newell, que permite "suavizar" las tasas brutas mediante el empleo de un denominador promedio que resulta de agregar el número de habitantes de las áreas de muy pequeño tamaño poblacional (25). Este grupo de análisis se realizó mediante el programa Clusterseer 2.10 (Biomedware Inc., Ann Arbor, MI, Estados Unidos).

CUADRO 1. Tasas de mortalidad infantil según el nivel de marginación social en el municipio Hermosillo, Sonora, México, 2000-2003

\begin{tabular}{|c|c|c|c|c|}
\hline $\begin{array}{l}\text { Área según el nivel de } \\
\text { marginación sociala }\end{array}$ & $\begin{array}{l}\text { Tasa de mortalidad } \\
\text { infantil }^{b}\end{array}$ & IC95\% ${ }^{c}$ & Razón de tasas ${ }^{d}$ & IC95\% \\
\hline General & 14,3 & $13,2-15,3$ & $N A^{e}$ & NA \\
\hline Muy bajo & 14,1 & $10,7-17,5$ & 1,2 & $1,0-1,4$ \\
\hline Bajo & 11,6 & $10,1-13,2$ & 1,0 & Referencia \\
\hline Medio & 14,6 & $12,4-16,9$ & 1,3 & $1,1-1,5$ \\
\hline Alto & 16,2 & $13,7-18,7$ & 1,4 & $1,2-1,7$ \\
\hline Muy alto & 14,7 & $10,9-18,5$ & 1,3 & $1,1-1,5$ \\
\hline \multicolumn{5}{|c|}{$\begin{array}{l}\text { a Según el índice de estatus socioeconómico, basado en la información del Censo Nacional de Población y Vivienda de } 2000 \\
\text { (23). } \\
\text { b Por } 1000 \text { nacidos vivos. } \\
\text { c IC95\%: intervalo de confianza de } 95 \% \text {. } \\
\text { d Exceso de riesgo de muertes infantiles en el área estudiada, calculada con respecto al área con bajo nivel de marginación } \\
\text { social, tomada como referencia. } \\
\text { e NA: no aplica. }\end{array}$} \\
\hline
\end{tabular}

La correlación espacial entre los IMU y las TMI "suavizadas" se analizó mediante el índice local bifactorial de asociación espacial (26). Se elaboró un mapa con este índice para identificar las AGEB de mayor riesgo dentro de la ciudad. Finalmente, se evaluó la relación entre las TMI y los IMU mediante un modelo de regresión lineal simple. Todos los valores de significación en los modelos de regresión y los mapas fueron de una cola y se calcularon mediante el programa de cómputo Geoda versión 0.9.5-i (Geoda Center, Arizona State University, Tempe, AZ, Estados Unidos). Se tomó como valor de significación $P \leq 0,05$.

Este estudio recibió la aprobación de los comités de ética de la Universidad de Michigan, Estados Unidos, y El Colegio de Sonora y la Secretaría de Salud Pública del Estado de Sonora, ambos de México.

\section{RESULTADOS}

En el período 2000-2003 se registraron 702 muertes infantiles en la ciudad de Hermosillo, para una TMI de 14,3 por 1000 nacidos vivos, ligeramente mayor que el promedio estatal (13,2 por 1000$)$. La tasa específica más alta (16,2 por $1000)$ correspondió al estrato residencial con alto nivel de marginación social, seguido por el de muy alta marginación $(14,7$ por 1000$)$; la menor tasa (11,6 por $1000)$ correspondió a las áreas de bajo nivel de marginación social. Comparados con este último estrato, se apreció un exceso del riesgo de muerte en los niños que residían en áreas de nivel medio de marginación, así como en las de marginalidad alta y muy alta (cuadro 1 ).
El valor del IMU en las AGEB, reflejo del nivel de marginación social, varió entre $-3,1$ y 6,6, con un promedio en las áreas de muy alta marginación de 4,4 y en las de menor marginación de $-2,7$, lo que demuestra la heterogeneidad en la distribución de los niveles de marginación dentro de la ciudad (cuadro 2). En general, $20(7,9 \%)$ de las 254 áreas residenciales se clasificaron como de muy alta marginación social y 38 (15,0\%) como de alta marginación. La mayoría de las áreas residenciales con niveles de marginación alta y muy alta se ubicaron en el noroeste, noreste y suroeste de la ciudad (figura 1). El índice de Moran I $(0,62)$ de esta distribución fue estadísticamente significativo $(P<0,001)$, lo que indica que había una autocorrelación espacial de la marginación.

La distribución espacial de las TMI suavizadas mostró áreas residenciales alejadas de la tasa media $(14,3$ por 1000$)$ en más de dos desviaciones estándar (figura 2). A pesar de la suavización, las tasas en algunas AGEB fueron inestables debido al reducido número de nacimientos registrados; no obstante, no se observó un patrón de agrupamiento espacial de las TMI, con valores no significativos del índice de Moran I $(-0,007$; $P=0,87)$ y del estadístico Ipop de Oden $(0,022 ; P=0,07)$. A pesar de esta falta de agrupamiento de las TMI en la ciudad, al aplicar la técnica de Besag y Newell se identificaron agrupamientos a escala local en ocho AGEB. Estos agrupamientos locales estuvieron principalmente ubicados en las zonas central y occidental de Hermosillo (figura 2). Esas áreas se pueden considerar pequeñas agrupaciones si la unidad de análisis es una sola 
CUADRO 2. Distribución de la marginación social por área geoestadística básica (AGEB) urbana del municipio Hermosillo, Sonora, México, 2000-2003

\begin{tabular}{|c|c|c|c|c|c|c|c|c|}
\hline \multirow{2}{*}{$\begin{array}{l}\text { Área según el nivel de } \\
\text { marginación sociala }\end{array}$} & \multicolumn{2}{|c|}{ AGEB } & \multicolumn{2}{|c|}{ Población } & \multicolumn{2}{|c|}{ Viviendas habitadas } & \multicolumn{2}{|c|}{$\begin{array}{c}\text { Índice de } \\
\text { marginación urbana }\end{array}$} \\
\hline & No. & $\%$ & No. & $\%$ & No. & $\%$ & Media & $\mathrm{DE}^{\mathrm{b}}$ \\
\hline General & 254 & 100 & 545928 & 100 & 133283 & 100 & 0 & 1,9 \\
\hline Muy bajo & 34 & 13,4 & 73141 & 13,4 & 18437 & 13,8 & $-2,7$ & 0,3 \\
\hline Bajo & 97 & 38,2 & 232721 & 42,6 & 58505 & 43,9 & $-0,8$ & 0,5 \\
\hline Medio & 39 & 15,3 & 99445 & 18,2 & 23600 & 17,7 & 0,4 & 0,3 \\
\hline Alto & 38 & 15,0 & 105455 & 19,3 & 24507 & 18,4 & 1,8 & 0,5 \\
\hline Muy alto & 20 & 7,9 & 31836 & 5,8 & 8114 & 6,1 & 4,4 & 1,1 \\
\hline Excluidos del análisis ${ }^{\mathrm{C}}$ & 26 & 10,2 & 3330 & 0,6 & 120 & 0,9 & NA & NA \\
\hline
\end{tabular}

a Según el índice de estatus socioeconómico, basado en la información del Censo Nacional de Población y Vivienda de 2000 (23).

b DE: desviación estándar.

${ }^{c}$ No se consideraron en el análisis por razones de confidencialidad, ya que tenían menos de 50 habitantes o menos de 20 viviendas habitadas.

FIGURA 1. Distribución de la marginación social según las áreas geoestadísticas básicas (AGEB) urbanas del municipio Hermosillo, Sonora, México, 2000-2003

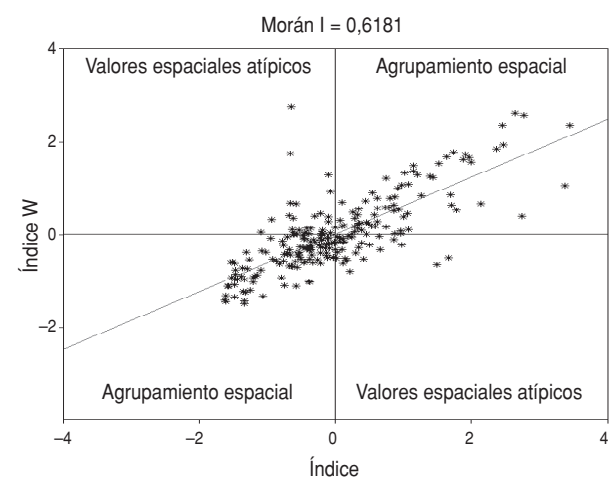

Nivel de marginación social

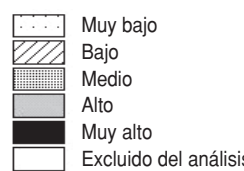

área o como agrupaciones de mayor tamaño si la unidad de análisis se conformara de varias áreas.

Según el mapa de la distribución espacial del índice local bifactorial de asociación espacial, las áreas presentaron autocorrelación local positiva, es decir, agrupamientos espaciales de los IMU y las TMI suavizadas (alto IMU-alta TMI; bajo IMU-baja TMI) (figura 3). En el mapa se observa una zona de alto riesgo (alto IMU-alta TMI) al noroeste de la ciudad y dos zonas de menor dimensión al este y sur de la ciudad. En el otro extremo se ubicó el agrupamiento espacial de las zonas central y noreste de la ciudad, en las que confluyen áreas de bajo nivel de marginación y de baja mortalidad infan-
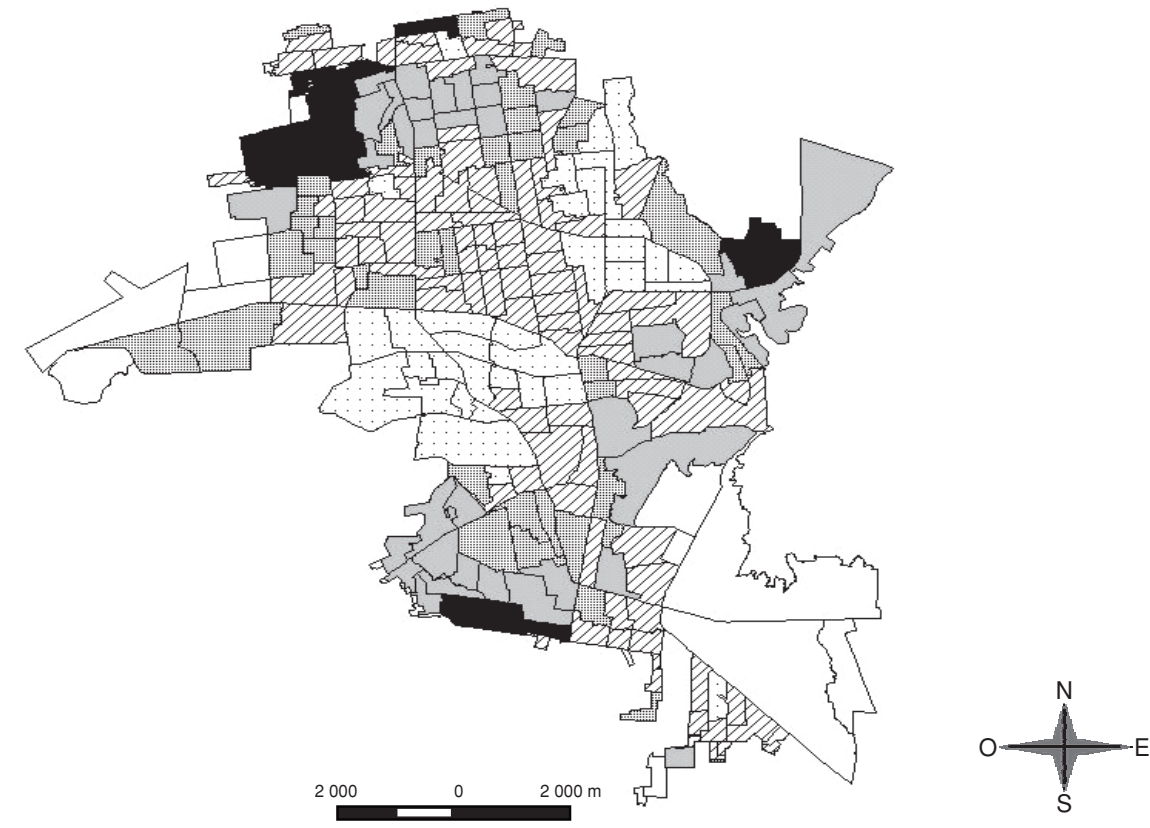

til. Este mapa también muestra la autocorrelación negativa existente en la zona central de la ciudad - lo que podría indicar la presencia de valores atípicos o outliers espaciales - con áreas de bajo nivel de marginación y alta mortalidad infantil. Mientras, en el suroeste se observaron áreas con baja TMI y alto IMU.

Finalmente, los resultados del análisis de regresión lineal indicaron que el nivel de marginación tiene un pequeño efecto sobre la mortalidad infantil, ya que no se observó significación estadística (cuadro 3). Sin embargo, en los modelos que examinaron el efecto individual de los indicadores socioeconómicos con los que se construyó el IMU sobre la distribución de la TMI, se apreció el efecto significa- tivo que tienen sobre la TMI el nivel educativo y el ingreso económico familiar.

\section{DISCUSIÓN}

Este estudio es un esfuerzo inicial dirigido a entender el efecto que tienen las disparidades socioeconómicas urbanas sobre la distribución espacial de la TMI en la ciudad de Hermosillo e intenta fortalecer la discusión sobre las desigualdades en salud en áreas urbanas del norte de México.

Se identificaron agrupamientos espaciales locales de áreas con altas TMI en el sector norte de Hermosillo. Se encontraron áreas con altos valores de IMU que se correlacionaron con áreas de elevadas 
FIGURA 2. Tasas de mortalidad infantil (TMI) suavizadas (izquierda) y agrupamiento espacial de las TMI (derecha) en las áreas geoestadísticas básicas urbanas del municipio Hermosillo, Sonora, México, 2000-2003

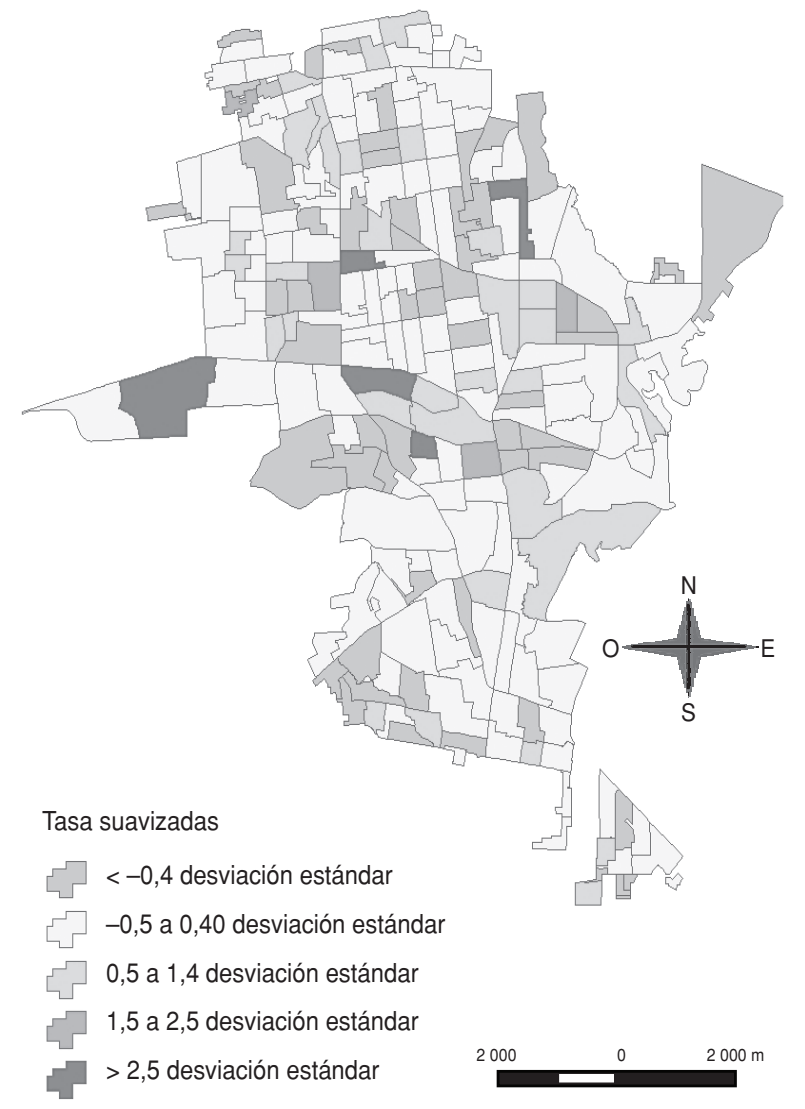

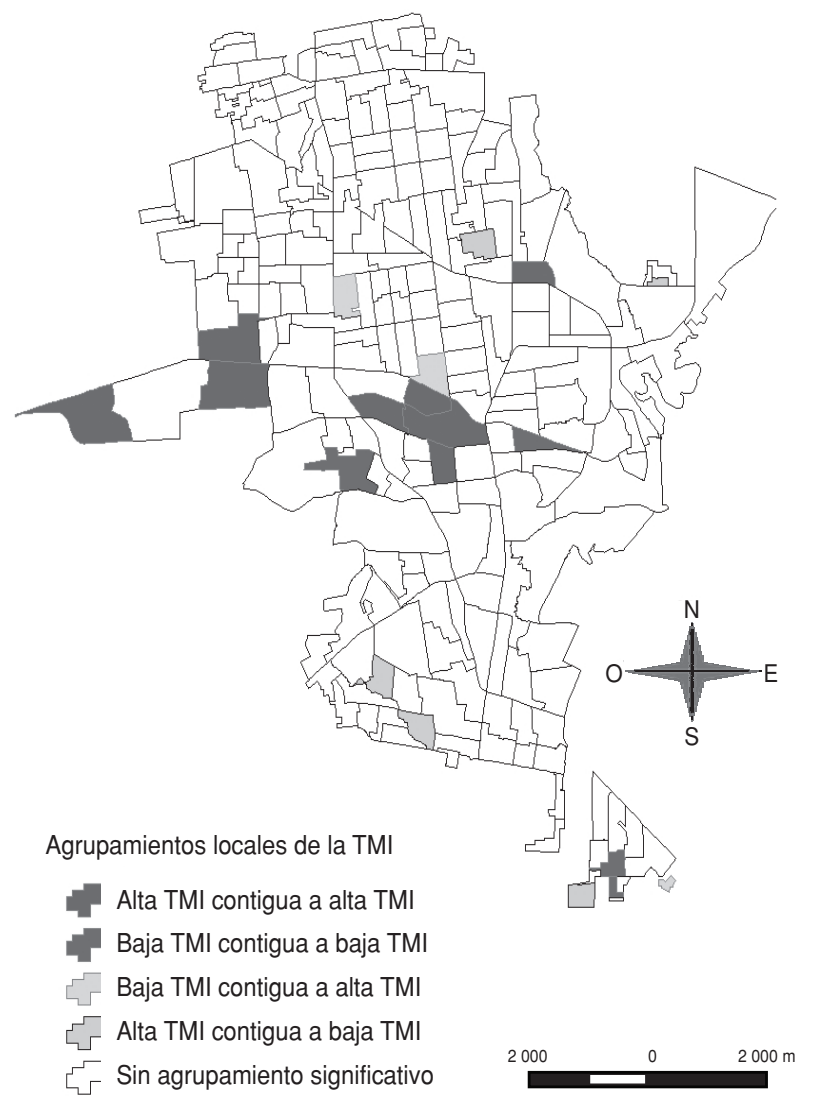

FIGURA 3. Agrupamiento bifactorial de los indicadores locales de asociación espacial a partir de la tasa de mortalidad infantil (TMI) ${ }^{\mathrm{a}}$ y su relación con el índice de marginación urbana, según las áreas geoestadísticas básicas urbanas del municipio Hermosillo, Sonora, México, 2000-2003

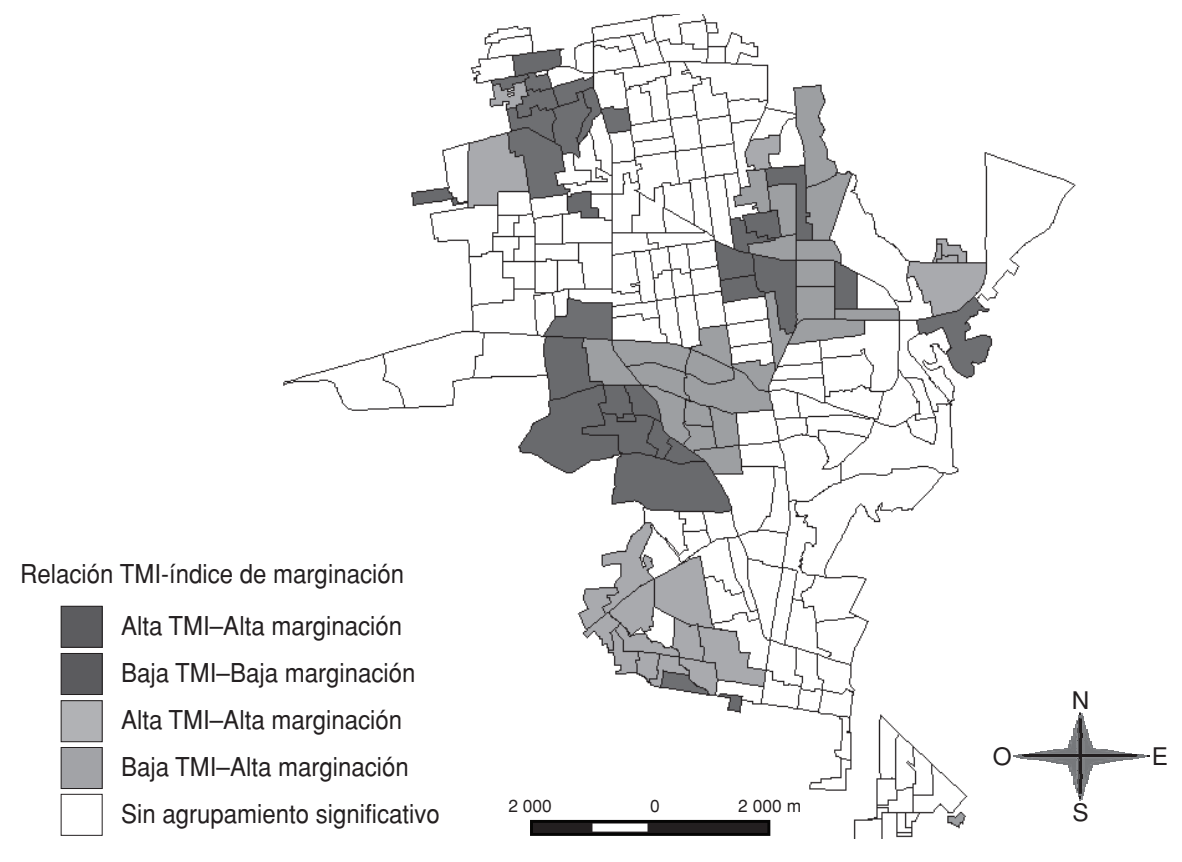

a Por 1000 nacidos vivos, suavizada por el procedimiento empírico de Bayes.
TMI, principalmente en el noroeste, aunque también en el noreste y suroeste de la ciudad. Estos resultados apoyan la hipótesis de que existe un efecto inverso del nivel socioeconómico sobre la distribución espacial de la mortalidad infantil en Hermosillo, ya que las áreas con mayores valores del IMU mostraron altas TMI. Este hallazgo es consistente con otras investigaciones realizadas en áreas geográficas pequeñas, regiones y países (27-29). En todos los casos se ha observado el efecto de la clase social y otras variables socioeconómicas —como los ingresos económicos y la educación de la madre-, independientemente de los abordajes conceptuales o los métodos empleados para analizar los factores socioeconómicos (30). Un hecho que llama la atención es que las AGEB con muy bajo nivel de marginación mostraron una mayor TMI que las que presentaban baja marginación. Esto podría deberse a que las áreas censales de muy baja marginación fueron las de menor tamaño poblacional, lo que puede haber generado una elevada inestabilidad en la va- 
CUADRO 3. Modelos finales de regresión lineal de las tasas de mortalidad infantil en el municipio Hermosillo, Sonora, México, 2000-2003

\begin{tabular}{|c|c|c|c|c|}
\hline Variable & Coeficiente $\beta^{a}$ & Error estándar & $t^{\mathrm{b}}$ & $P^{b}$ \\
\hline \multicolumn{5}{|l|}{ Modelo $1^{\mathrm{c}}$} \\
\hline Intersección & 15,2 & 0,11 & 13,71 & 0,00 \\
\hline Índice de marginación & $-0,42$ & 0,58 & $-0,72$ & 0,47 \\
\hline \multicolumn{5}{|l|}{ Modelo $2^{d}$} \\
\hline Intersección & 22,0 & 12,18 & 1,81 & 0,07 \\
\hline Población $\geq 15$ años sin educación mayor de primaria, \% & 0,37 & 0,18 & 2,08 & 0,04 \\
\hline Viviendas con paredes construidas con materiales precarios, $\%$ & 0,00 & 0,00 & 0,40 & 0,69 \\
\hline Familias que no poseen carro propio, \% & $-0,11$ & 0,16 & $-0,69$ & 0,49 \\
\hline Viviendas con hacinamiento (un solo cuarto para dormir), \% & 0,12 & 0,12 & 0,97 & 0,33 \\
\hline Población $\geq 15$ años que gana de 0 a 2 salarios mínimos, $\%$ & $-0,39$ & 0,15 & $-2,60$ & 0,01 \\
\hline Viviendas con drenaje y electricidad, \% & 0,02 & 0,10 & 0,23 & 0,82 \\
\hline Población con seguro de salud, \% & $-0,06$ & 0,14 & $-0,48$ & 0,64 \\
\hline
\end{tabular}

a Cambio promedio en la tasa de mortalidad infantil.

b Según la prueba de la $t$ de Student para diferencia de medias.

c $F=0,526285 ; P=0,47$.

d $F=2,02227 ; P=0,05$.

rianza de las TMI, no controlada mediante el enfoque estadístico empleado.

La identificación de agrupamientos espaciales con alto IMU y alta TMI en áreas específicas de la ciudad puede obedecer, por un lado, a que en esas áreas confluyen la pobreza y la migración, lo que genera un creciente volumen de nuevas áreas residenciales de bajo nivel socioeconómico (conocidas popularmente como "invasiones"). Por otro lado, es posible que la relativa lejanía de esos vecindarios con respecto a los principales hospitales de la ciudad también influya negativamente en la mortalidad, algo ya demostrado por otros autores (31). Se requieren nuevos estudios para confirmar esas hipótesis en Hermosillo y otras ciudades similares. Aunque no se encontró un efecto significativo de los IMU sobre la distribución espacial de las TMI, es posible que esto se deba a que no existe una relación lineal entre estos indicadores.

La capacidad confirmada de los SIG de desplegar la distribución espacial de factores de riesgo y eventos de salud (32-34) puede contribuir a dar la prioridad requerida a intervenciones de salud pública, particularmente las dirigidas a las poblaciones vulnerables. No obstante su valor, se subraya que la capacidad tecnológica de los SIG requiere el complemento de un análisis estadístico apropiado (35-37). Como ejemplo de ello, en un primer mapa (no mostrado) en el que se desplegaron tasas no ajustadas de mortalidad se observaron TMI muy elevadas en AGEB en las que ocurrieron muy pocos nacimientos; si se hubiera pasado por alto la inestabilidad inherente a la varianza (desigual precisión) de este hecho se hubiera concluido que había una correlación espacial, aunque en realidad no existiera.

El poder visual de los mapas producidos con ayuda de los SIG debe complementarse con el empleo de técnicas estadísticas de análisis espacial, como la de Besag y Newell (25) que permitió detectar agrupamientos locales de elevadas TMI en algunas áreas del norte de la ciudad, a pesar de la heterogeneidad del tamaño poblacional de las AGEB. Esta técnica es muy útil cuando se analizan áreas de pequeño tamaño poblacional, como son las colonias o los barrios de ciudades medianas y pequeñas, como Hermosillo.

Se confirmó la utilidad del índice local bifactorial de asociación espacial para examinar la correlación entre dos variables de grupo. Este procedimiento permitió identificar áreas residenciales con alto riesgo de mortalidad infantil dentro de la ciudad. Para asignarlas a esa categoría de riesgo, estas áreas no solo debían contar con elevadas TMI, sino también debían estar rodeadas por áreas con alto grado de marginación social. Esto se logró gracias a que este índice utiliza una matriz de adyacencia de primer orden (áreas geográficas que comparten una frontera con otra área de interés) para evaluar el grado de agrupamiento espacial. La importancia de identificar este tipo de agrupamiento radica en que la vecindad geográfica no solo propicia que se compartan ciertos atributos residenciales, sino también algunos factores de riesgo y patrones de daños a la salud. De ahí la conveniencia de considerar cuidadosamente las correlaciones espaciales subyacentes para evitar inferencias erróneas (38).

El empleo del índice local de asociación espacial, sea simple o bifactorial, puede contribuir a establecer el grado de priorización de los problemas de salud y ayudar a los encargados de tomar decisiones a dirigir intervenciones de salud a las áreas con mayor vulnerabilidad social dentro de los espacios urbanos. Esto es de particular interés para el caso de México, pues la distribución espacial de las TMI se ha documentado solamente mediante el estudio de grandes regiones geográficas, como son los municipios o las provincias (39-40), pero no para examinar la distribución de los eventos relacionados con la salud en el interior de las ciudades.

No obstante lo anterior, cuando las unidades de análisis son áreas censales o barrios es difícil controlar la autocorrelación espacial residual generada por la inestabilidad de la varianza de los indicadores examinados. Esto puede provocar distorsiones en los mapas producidos con ayuda de los SIG (18). Por ello se sugiere que en investigaciones futuras sobre este tema en ciudades medianas y pequeñas se empleen enfoques estadísticos más integrales, como el modelaje jerárquico bayesiano, los modelos de Poisson y los modelos espaciales de efectos aleatorios.

Independientemente del abordaje estadístico que se seleccione, un desafío operativo en los estudios que se lleven a cabo en este rubro en México será mejorar la estabilidad de los estimadores de riesgo (por ejemplo, la incidencia de enfermedades y la mortalidad), de modo que, aunque la unidad de análisis sea pequeña, se garantice la confiabilidad del estimador. Algunas alternativas que se pueden recomendar para estos estudios son: a) agregar los datos de las áreas censales me- 
diante criterios de contigüidad geográfica y semejanza socioeconómica, de modo que aumente el tamaño poblacional de cada área de análisis; b) aumentar los límites administrativos del área en estudio (como áreas postales, distritos administrativos, distritos electorales y jurisdicciones sanitarias); y c) alargar el período de estudio. En todo caso, el desafío metodológico impuesto por el uso de nuevas unidades de análisis o períodos de estudio ha sido objeto de discusión y se ha propuesto convertir los datos censales en diferentes sistemas de zona (41). Estas opciones pueden explorarse en nuevos estudios epidemiológicos de este tipo.

Otro asunto que amerita particular atención en futuros estudios de análisis espacial es el empleo de datos de buena calidad para localizar los eventos analizados, pues de otro modo puede llegarse a interpretaciones erróneas (42-43). En el presente estudio se confrontaron dificultades para completar los datos necesa- rios, fundamentalmente debido a la ausencia o el subregistro de la información domiciliaria y de otras variables socioeconómicas en las bases de datos empleadas. De hecho, no se contó con los datos necesarios para ubicar $5,1 \%$ de las defunciones registradas, por lo que hubo que asignarles un área mediante criterios que podrían introducir un sesgo en la clasificación. Esta falta de información también pudo haber afectado la precisión de los denominadores al estimar las tasas en las AGEB.

Como conclusión, en este estudio se encontraron agrupamientos espaciales con altas TMI en áreas socialmente marginadas del noroeste de Hermosillo, una ciudad de tamaño medio ubicada en el noroeste de México. Estos resultados, obtenidos mediante la combinación de técnicas de análisis espacial y herramientas de los SIG pueden ayudar a dirigir intervenciones específicas hacia esas áreas residenciales de alto riesgo. Los hallaz- gos demuestran que el análisis espacial es una estrategia valiosa para comprender la distribución espacial de eventos de salud y sus factores de riesgo. El mejoramiento de la calidad de los registros de mortalidad infantil y nacimientos en Hermosillo podría reducir los efectos negativos de sesgos y mejorar la calidad del análisis espacial.

Agradecimientos. Este estudio recibió financiamiento de los Institutos Nacionales de Salud de los Estados Unidos de América (NIH Research Grant \#D43 TW001276), el Centro Internacional Fogarty y Office of Behavioral and Social Science Research (R21 TW06489). Se agradece el apoyo de la Secretaría de Salud Pública y de la Oficialía del Registro Civil, ambas del Estado de Sonora, así como de la delegación estatal del Instituto Nacional de Estadística, Geografía e Informática, por facilitar los datos empleados en el análisis.

\section{REFERENCIAS}

1. Ordorica-Mellado M. Cambios demográficos y desafíos para la política de población en México. Una reflexión a largo plazo. Papeles de población. Toluca, México: Universidad Autónoma del Estado de México; 2004. Hallado en http://redalyc.uaemex.mx/redalyc/pdf/ 112/11204003.pdf. Acceso el 26 de junio de 2009.

2. Sepúlveda J, Bustreo F, Tapia R, Rivera J, Lozano R, Olaíz G, et al. Aumento de la sobrevida en menores de cinco años en México: la estrategia diagonal. Salud Publica Mex. 2007; 49(Supl1):S110-5.

3. Aguilera N, Marrufo GM. Can better infrastructure and quality reduce hospital infant mortality rates in Mexico? Health Policy. 2007;80:239-52.

4. Rangel-Gómez G, González-Ramírez RS. La situación de la salud en la frontera norte. En: Ordóñez-Barba G, Reyes-Santos MS, coord. Los retos de la política social en la frontera norte de México. Tijuana, México: El Colegio de la Frontera Norte, Plaza y Valdez; 2006. Pp. 181-211.

5. Denman C, Lara F, DeZapien J. Salud pública, medio ambiente y participación social en un contexto fronterizo. En: Haro JA, de Keijzer B, coord. Participación comunitaria en salud: evaluación de experiencias y tareas para el futuro. Hermosillo, México: El Colegio de Sonora, Produssep AC, Organización Panamericana de la Salud; 1998. Pp. 244-77.

6. Kopinak K, Barajas R. Too close for comfort? The proximity of industrial hazardous wastes to local populations in Tijuana, Baja California. J Environ Dev. 2002;11:215-46.
7. García H, Lara F. Repas ambulants; informalité urban et modernité industrielle á la frontiére du Mexique. Sociol Trav. 2004;46:42-53.

8. Denman C. Local response to global development: an emerging culture of health among pregnant women in Mexican maquiladoras. En: Grown C, Braunstein E, Malhotra A, eds. Trading women's health and rights? Trade liberalization and reproductive health in developing economies. New York: Zed Books; 2006. Pp. 143-63.

9. Harlow S, Denman C, Cedillo L. Occupational and population health profiles: a public health perspective on the social costs and benefits of export-led development. In: Kopinak K, ed. The social costs of industrial growth in northern Mexico. Boulder, CO: Lynne Rienner; 2005. Pp. 133-78.

10. Loyola E, Castillo-Salgado C, Nájera-Aguilar P, Vidaurre M, Mujica OJ, Martínez-Piedra R. Los sistemas de información geográfica como herramienta para monitorear las desigualdades de salud. Rev Panam Salud Pública. 2002; 12(6):415-28.

11. Reidpath DD, Allotey P. Infant mortality rate as an indicator of population health. J Epidemiol Community Health. 2003;57:344-6.

12. Stockwell EG. Infant mortality and socioeconomic status: a changing relationship. Milbank Mem Fund Q. 1962;40(1):101-11.

13. Madise NJ, Banda EM, Benaya KW. Infant mortality in Zambia: socioeconomic and demographic correlates. Soc Biol. 2003;(1/2):148-66.

14. Hosseinpoor AR, Van Doorslaer E, Speybroeck N, Naghavi M, Mohammad K, Majdzadeh $\mathrm{K}$, et al. Decomposing socioeconomic inequality in infant mortality in Iran. Int J Epidemiol. 2006;35:1211-9.

15. Alves-Monteiro R, de Abreu Soares Schmitz B. Infant mortality in the Federal District, Brazil: time trends and socioeconomic inequalities. Cad Saude Publica. 2007;23(4):767-74.

16. Singh GK, Kogan MD. Widening socioeconomic disparities in US childhood mortality, 1969-2000. Am J Public Health. 2007;97(9): 1658-65.

17. Pickle LW. Mapping mortality data in the United States. In: Elliot P, Wakefield J, Best N, Briggs D. Spatial epidemiology: methods and applications. 1st Ed. Oxford: Oxford University Press; 2000. Pp. 240-50.

18. Anselin L, Kim YW, Syabri I. Web-based spatial analytical tools for the exploration of spatial data. J Geograph Syst. 2004;6(2):197-218.

19. Phillips J. An analysis of infant mortality in the District of Columbia using geographic information systems as a data integration tool. Hallado en http://www.dchealth.dc.gov/ $\mathrm{DOH} /$ frames.asp?doc $=/ \mathrm{doh} / \mathrm{lib} / \mathrm{doh} /$ services/administration_offices/schs/pdf/ analysis_of_infant_mortality_gis.pdf. Acceso el 16 de junio de 2009.

20. Instituto Nacional de Estadística, Geografía e Informática. Segundo Conteo de Población y Vivienda 2005. Aguascalientes: INEGI; 2006.

21. Instituto Nacional de Estadística, Geografía e Informática. Principales resultados del XII Censo General de Población y Vivienda 2000. Aguascalientes: INEGI; 2001.

22. Hijar M, Trostle J, Bronfman M. Pedestrian injuries in Mexico: a multi-method approach. Soc Sci Med. 2003;57:2149-59. 
23. Estados Unidos Mexicanos, Consejo Nacional de Población. Índice de marginación a nivel estatal y municipal. Concepto y dimensiones de la marginación. Metodología de estimación del índice de marginación. México, D.F.: CNP; 2000.

24. Oden N. Adjusting Moran's I for population density. Stat Med. 1995;14:17-26.

25. Besag J, Newell J. The detection of clusters in rare diseases. J Royal Statist Soc Series A. 1991;154:143-55.

26. Anselin L, Syabri I, Kho Y. Geoda: an introduction to spatial data analysis. Geogr Anal. 2006;38:5-22. Hallado en http://www.geo vista.psu.edu/publications/2006/Anselin GeoDa.pdf. Acceso el 26 de junio de 2009.

27. Congdon $P$, Southall H. Small area variations in infant mortality in England and Wales in the inter-war period and their link with socioeconomic factors. Health Place. 2004;10:363-82.

28. Hertel-Fernández AW, Giusti AE, Sotelo JM. The Chilean infant mortality decline: improvement for whom? Socioeconomic and geographic inequalities in infant mortality, 1990-2005. Bull World Health Organ. 2007; 85(10):798-804.

29. Wagstaff A. Socioeconomic inequalities in child mortality: comparison across nine developing countries. Bull World Health Organ. 2000;78:19-29.
30. Lachaud JP. Modelling determinants of child mortality and poverty in the Comoros. Health Place. 2004;10:13-42.

31. Feudtner C, Silveira MJ, Shabbout M, Hoskins RE. Distance from home when death occurs: a population-based study of Washington State, 1989-2002. Pediatr. 2006;117:e932-9.

32. Moore D, Carpenter T. Spatial analytical methods and geographic information systems: use in health research and epidemiology. Epidemiol Rev. 1999;21(2):143-61.

33. Glass G. Spatial aspects of epidemiology: the interface with medical geography. Epidemiol Rev. 2000;22(1):136-9.

34. Cromley E, McLafferty S. GIS and public health. 1st ed. New York: Guilford Press; 2002.

35. Haining R. Exploratory spatial data analysis: numerical methods. In: Spatial data analysis. Theory and practice. Cambridge: Cambridge University Press; 2003. Pp. 242-6.

36. Marshall TJ. A review of methods for the statistical analysis of spatial patterns of disease. J Royal Statist Soc A. 1991;154(3):421-41.

37. Unwin A, Unwin D. Exploratory spatial data analysis with local statistics. Statistician. 1998;47(3):415-21.

38. Richardson S, Monfort C. Ecological correlation studies. In: Elliot P, Wakefield J, Best N, Briggs D. Spatial epidemiology: methods and applications. 1st ed. Oxford: Oxford University Press; 2000. Pp. 205-20.

39. Méndez-González RM, García de Fuentes A, Cervera-Montejano MD. Mortalidad infantil y marginación social en la Península de Yucatán. Inv Geogr Bol. 2004;54:140-63.

40. Gutiérrez JP, Bertozzi SM. La brecha en salud en México medida a través de la mortalidad infantil. Salud Publica Mex. 2003;45:102-9.

41. Briggs D, Fletch D, de Hoog K. Census data issues for epidemiology and health risk assessment: experiences from the small area health statistics unit. J Royal Statist Soc A. 2007;170 (Part 2): 355-78.

42. Krieger N, Waterman P, Lemieux K, Zierler S, Hogan JW. On the wrong side of the tracts? Evaluating the accuracy of geocoding in public health research. Am J Public Health. 2001; 91:1114-6.

43. Forand SP, Talbot TO, Druschel C, Cross PK. Data quality and the spatial analysis of disease rates: congenital malformations in New York State. Health Place. 2002;8:191-9.

Manuscrito recibido el 20 de enero de 2008. Aceptado para publicación, tras revisión, el 1 de agosto de 2008.

ABSTRACT Objective. To identify areas with high risk of infant mortality and any possible correlation with the population's socioeconomic status through the use of a geographic information system and spacial analysis techniques.

Infant mortality and urban marginalization: a spatial analysis of their relationship in a medium-sized city in northwest Mexico

Methods. An exploratory ecologic study was conducted in Hermosillo, the capital of Sonora, Mexico, in 2000-2003. The urban marginalization index (UMI) and the infant mortality rate (IMR) were determined for each of the city's basic geostatistical areas (BGA). The UMI and IMR were statistically calculated to identify geographic areas in which they were concentrated and to determine the degree of spatial correlation between these indicators. To determine the general spatial autocorrelation and spatial clustering of UMIs and IMRs within the city and the BGAs, Morans I index, Ipop statistics, and Besag and Newell's method were employed.

Results. The mean IMR was 14.3 per 1000 live births, higher in the BGAs with greater social marginalization (16.2 per 1000$)$ and lower in those with less (11.7 per 1000 ). The UMI range was -3.1-6.6 (maximum: 4.3; minimum: -2.7 ). Autocorrelation was found among the UMI (Moran I = 0.62), with significant clustering in the city's northwest, northeast, and southeast parts. Local clustering of high IMRs was found in Hermosillo's central and western areas, albeit without autocorrelation (Moran $\mathrm{I}=-0.007$ ). High risk areas (high IMR and high UMI) were found in the city's northwestern section.

Conclusions. Spatial clusters with high IMR were found in socially marginalized areas in the northwestern part of Hermosillo, a city of medium size located in northwestern Mexico. These results, reached through a combination of spatial analysis techniques and geographic information tools can help guide interventions specifically designed for these high risk residential areas.

Key words Infant mortality, socioeconomic factors, geographic localization of risk, Mexico. 